\title{
Obesity, walking pace and risk of severe COVID-19 and mortality: analysis of UK Biobank
}

\author{
Thomas Yates $\mathbb{1}^{1,2}$. Cameron Razieh ${ }^{1,2}$ - Francesco Zaccardi ${ }^{1,3} \cdot$ Alex V. Rowlands $\mathbb{1}^{1} \cdot$ Samuel Seidu ${ }^{1}$. \\ Melanie J. Davies ${ }^{1,2,4} \cdot$ Kamlesh Khunti $\mathbb{1}^{1,3,4,5}$
}

Received: 17 June 2020 / Revised: 11 December 2020 / Accepted: 21 January 2021 / Published online: 26 February 2021

(c) The Author(s), under exclusive licence to Springer Nature Limited 2021

\begin{abstract}
Obesity is an emerging risk factor for coronavirus disease-2019 (COVID-19). Simple measures of physical fitness, such as self-reported walking pace, may also be important risk markers. This analysis includes 412,596 UK Biobank participants with linked COVID-19 data (median age at linkage $=68$ years, obese $=24 \%$, median number of comorbidities $=1$ ). As of August 24th 2020, there were 1001 cases of severe (in-hospital) disease and 336 COVID-19 deaths. Compared to normal weight individuals, the adjusted odds ratio (OR) of severe COVID-19 in overweight and obese individuals was 1.26 (1.07, $1.48)$ and $1.49(1.25,1.79)$, respectively. For COVID-19 mortality, the ORs were $1.19(0.88,161)$ and $1.82(1.33,2.49)$, respectively. Compared to those with a brisk walking pace, the OR of severe COVID-19 for steady/average and slow walkers was $1.13(0.98,1.31)$ and $1.88(1.53,2.31)$, respectively. For COVID-19 mortality, the ORs were $1.44(1.10,1.90)$ and $1.83(1.26,2.65)$, respectively. Slow walkers had the highest risk regardless of obesity status. For example, compared to normal weight brisk walkers, the OR of severe disease and COVID-19 mortality in normal weight slow walkers was 2.42 $(1.53,3.84)$ and $3.75(1.61,8.70)$, respectively. Self-reported slow walkers appear to be a high-risk group for severe COVID19 outcomes independent of obesity.
\end{abstract}

\section{Introduction}

The severe acute respiratory syndrome coronavirus 2 (SARS-CoV-2), which causes coronavirus disease-2019 (COVID-19), is devastating global economies and putting unprecedented strain on health care services and communities. The mortality and economic burden caused by the

$\triangle$ Thomas Yates

ty20@le.ac.uk

1 Diabetes Research Centre, University of Leicester, Leicester General Hospital, Leicester, UK

2 National Institute for Health Research (NIHR) Leicester Biomedical Research Centre (BRC), NIHR Leicester Biomedical Research Centre, University of Leicester, Leicester, UK

3 Leicester Real World Evidence Unit, Diabetes Research Centre, University of Leicester, Leicester, UK

4 Leicester Diabetes Centre, University Hospitals of Leicester, Leicester General Hospital, Leicester, UK

5 NIHR Applied Research Collaboration - East Midlands (ARCEM), University of Leicester, Leicester, UK virus have led to global research efforts to identify people at greatest risk of developing severe illness [1].

Obesity has emerged as a key risk factor for COVID-19 outcomes [2], with excess ectopic fat accumulation associated with higher chronic sub-clinical inflammation, functional immunologic deficit and a pro-thrombotic state potentially explaining the higher rates of disseminated intravascular coagulation and thromboembolism in severe COVID-19 patients [3]. Frailty has also been identified as a potential risk factor for severe COVID-19 [4], with early research showing it to be a stronger predictor of COVID-19 mortality than either age or comorbidities [5]. However, the interaction between measures of obesity and frailty in the association with COVID-19 outcomes has not been investigated.

Walking pace is a central indicator of whole-body physical fitness, frailty, reserve and resilience [6], with selfreported walking pace consistently found to be strongly associated with cardiorespiratory fitness, cardiovascular mortality and all-cause mortality, along with being a powerful marker of longevity [6-10]. Indeed, self-reported walking pace has been shown to be a stronger predictor of cardiovascular mortality than other measures of physical activity or function $[9,10]$, with self-reported slow walkers 
having a low life expectancy even in those with normal weight [8]. These findings suggest that self-reported walking pace acts as a simple easy to collect measure of overall physical health status. However, although well researched with cardiovascular and all-cause mortality outcomes, this hypothesis has not been established for infectious disease.

The aim of this brief report is to examine the relative association of body mass index (BMI) and self-reported walking pace with the risk of severe COVID-19 and COVID-19 mortality. We hypothesise that self-reported slow walkers would have poorer outcomes across all categories of obesity status.

\section{Methods}

For this analysis, we used UK Biobank (https://www.ukbioba nk.ac.uk/), a large prospective cohort of middle-aged adults designed to support health research [11]. Between March 2006 and July 2010, 502,543 individuals living within 25 miles of one of the 22 study assessment centres located throughout England, Scotland and Wales were recruited and provided comprehensive data on a broad range of factors. Ethical approval was obtained from the North West Centre for Research Ethics Committee (Ref: 11/NW/0382); all participants provided informed consent. UK Biobank data has been linked to Public Health England's Second Generation Surveillance System for national SARS-CoV-2 laboratory test data collected throughout England [12]. Data provided included specimen date and origin (hospital inpatient vs other). Data were available for the period 16th March 2020 to August 24th 2020. We restricted the analysis to those from English centres and alive as of 16th March and thus covered by the linkage system. We have classified a positive test result from an in-hospital setting as defining severe COVID-19, consistent with the detailed linkage information provided for this dataset [12]. COVID-19 mortality was defined where an ICD-10 code of U07.1 or U07.2 was listed as the primary cause of death on the death certificate. Mortality data was available up to 24th August 2020.

BMI and walking pace were collected at UK Biobank baseline assessment. BMI was categorised as normal weight $\left(18.5-24.9 \mathrm{~kg} / \mathrm{m}^{2}\right)$, overweight $\left(25-29.9 \mathrm{~kg} / \mathrm{m}^{2}\right)$, and obese $\left(\geq 30 \mathrm{~kg} / \mathrm{m}^{2}\right)$. Underweight individuals $\left(\mathrm{BMI}<18.5 \mathrm{~kg} / \mathrm{m}^{2}\right)$ were removed from the analysis due to insufficient cases. Habitual walking pace was self-reported as slow $(<3 \mathrm{mph})$, steady/average $(3-4 \mathrm{mph})$, or brisk $(>4 \mathrm{mph})$ and has been shown to be associated with cardiorespiratory fitness [6]. Mutually exclusive categories of walking pace and obesity status were created to investigate the odds of severe disease and COVID-19 mortality across possible combinations of these factors.
Medical history, including prevalent illnesses, were assessed by interview during baseline visit; ethnicity was self-reported and social deprivation defined by the Townsend Index (a composite measure of deprivation based on unemployment, non-car ownership, non-home ownership, and household overcrowding; negative values represent less deprivation). Age was calculated at March 16th 2020, the first available day for linkage used in this analysis.

We undertook logistic regression to analyse the association of obesity status, walking pace and their combination with the risk of severe disease or COVID-19 mortality. Analysis was based on a whole population level approach as described previously for UK Biobank [13], with severe disease or COVID-19 mortality cases compared to the remaining UK Biobank population. Models were adjusted for age, sex, ethnicity, social deprivation, number of reported illnesses per person, and the follow-up time from baseline to data collection. Data are reported as odds ratios (OR) with 95\% CI and results are deemed significant at $p<$ 0.05 (i.e., $95 \% \mathrm{CI}$ does not cross the dull).

\section{Results}

There were 412,596 individuals that were alive from English centres with complete covariate data, and thus eligible for coverage by the data linkage system. In total, there were 1001 cases of severe disease and 336 COVID-19 related deaths. The characteristics of the included participants, stratified by BMI categories, are reported in Table 1 .

Both BMI and walking pace were independently associated with the risk of severe disease and COVID-19 mortality. Compared to normal weight individuals, the adjusted OR of severe COVID-19 in overweight and obese individuals was $1.26(1.07,1.48)$ and $1.49(1.25,1.79)$, respectively. For COVID-19 mortality, the OR for same comparisons were $1.19(0.88,161)$ and $1.82(1.33,2.49)$, respectively.

Compared to those with a brisk walking pace, the OR of severe COVID-19 for steady/average and slow walkers was $1.13(0.98,1.31)$ and $1.88(1.53,2.31)$, respectively. For COVID-19 mortality, the OR for same comparisons were $1.44(1.10,1.90)$ and $1.83(1.26,2.65)$, respectively.

The odds of severe disease and COVID-19 mortality across combinations of obesity status and walking pace are shown in Fig. 1. Compared to normal weight brisk walkers, the odds of severe disease or COVID-19 mortality was highest in slow walkers. For example, compared to normal weight brisk walkers, the odds of severe COVID-19 in obese brisk walkers was $1.39(0.98,1.96)$, whereas the odds in normal weight slow walkers was $2.42(1.53,3.84)$. Similarly, compared to normal weight brisk walkers, the odds of COVID-19 mortality in obese brisk walkers was 
Table 1 Participant characteristics stratified by obesity status.

\begin{tabular}{|c|c|c|c|c|c|c|c|c|c|}
\hline & \multicolumn{3}{|l|}{$\begin{array}{l}\text { Normal weight } \\
(n=137,340)\end{array}$} & \multicolumn{3}{|c|}{$\begin{array}{l}\text { Overweight } \\
(n=176,519)\end{array}$} & \multicolumn{3}{|c|}{$\begin{array}{l}\text { Obese } \\
(n=98,737)\end{array}$} \\
\hline & $\mathrm{N}$ & \multicolumn{2}{|l|}{$\%$} & $\mathrm{~N}$ & \multicolumn{2}{|l|}{$\%$} & $\mathrm{~N}$ & \multicolumn{2}{|l|}{$\%$} \\
\hline \multicolumn{10}{|l|}{ Outcome } \\
\hline Severe COVID-19 cases & 223 & 0.16 & & 431 & 0.24 & & 347 & 0.35 & \\
\hline COVID-19 related deaths & 63 & 0.05 & & 137 & 0.08 & & 136 & 0.14 & \\
\hline \multicolumn{10}{|l|}{ Categorical factors } \\
\hline \multirow[t]{2}{*}{ Sex } & Female & 90287 & 66 & & 84048 & 48 & & 52879 & 54 \\
\hline & Male & 47053 & 34 & & 92471 & 52 & & 45858 & 46 \\
\hline \multirow[t]{2}{*}{ Ethnicity } & White European & 129808 & 95 & & 165925 & 94 & & 91861 & 93 \\
\hline & Other & 7532 & 5 & & 10594 & 6 & & 6876 & 7 \\
\hline \multirow[t]{3}{*}{ Walking Pace } & Brisk & 73918 & 54 & & 69135 & 39 & & 20859 & 21 \\
\hline & Steady/average & 59063 & 43 & & 97469 & 55 & & 62033 & 63 \\
\hline & Slow & 4359 & 3 & & 9915 & 6 & & 15845 & 16 \\
\hline Continuous factors & Median & 25th & 75th & Median & 25th & 75th & Median & 25th & 75th \\
\hline Age (years) & 67 & 60 & 73 & 69 & 61 & 74 & 69 & 61 & 74 \\
\hline Social deprivation (Townsend score) & -2.31 & -3.72 & 0.16 & -2.28 & -3.70 & 0.20 & -1.74 & -3.40 & 1.24 \\
\hline Diseases or illnesses (n per person) & 1 & 0 & 2 & 1 & 1 & 3 & 2 & 1 & 3 \\
\hline
\end{tabular}

25 th and 75 th indicate 25 th and 75 th percentile, respectively.

\section{Severe COVID-19}

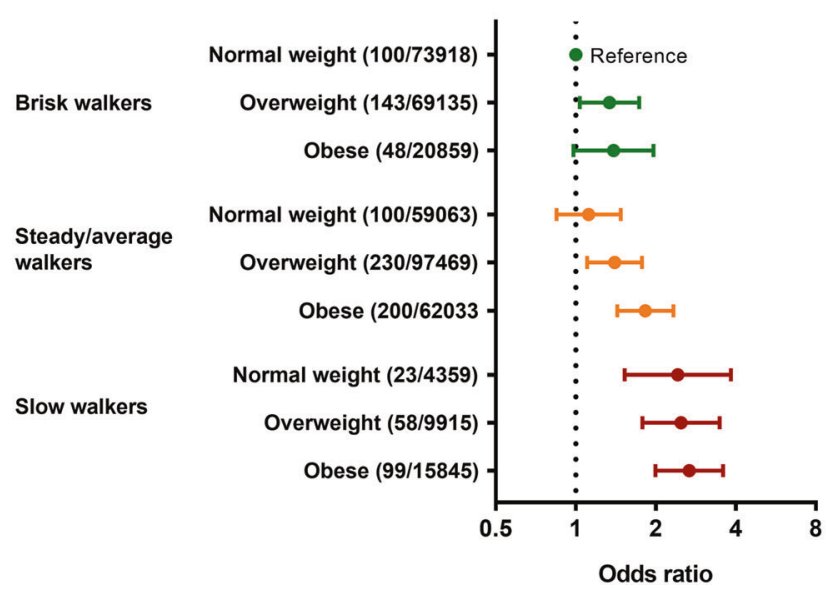

Fig. 1 Association of combined obesity and walking pace categories with severe COVID-19 and COVID-19 mortality. Bars indicate $95 \% \mathrm{CI}$; numbers in brackets are cases/total number in the

$2.58(1.33,5.02)$ whereas the odds in normal weight slow walkers was $3.75(1.61,8.70)$.

\section{Discussion}

Both obesity and self-reported walking pace are independently associated with the population level risk of severe COVID-19 and COVID-19 mortality in UK Biobank. However, self-reported slow walkers had the highest risk regardless of their obesity status, with normal weight slow

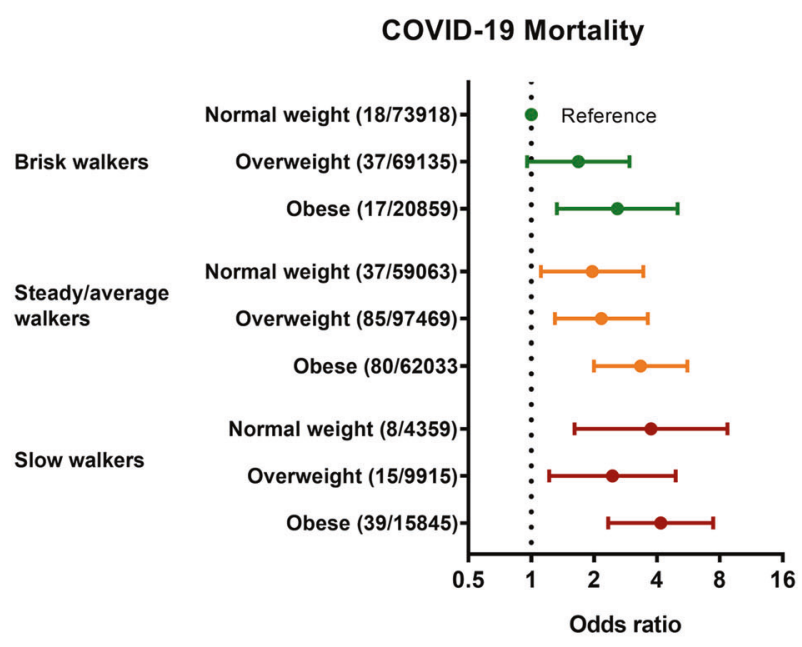

specific group. Adjusted for age, sex, ethnicity, number of illnesses, social deprivation, and follow-up time from baseline assessment.

walkers having over twice the risk of severe disease and almost four times the risk of COVID-19 mortality compared to normal weight brisk walkers.

Large routine database studies have reported the association of obesity with COVID-19 outcomes [14], with mechanisms hypothesised to include restricted pulmonary function and chronic inflammation [2, 3], with adipose tissue further suggested as an important vial reservoir [15]. However, routine clinical databases do not have data on measures of physical function or fitness. Findings for this study are consistent with those for all- 
cause and cardiovascular mortality outcomes where selfreported slow walkers have an elevated risk of cardiovascular mortality or early death, particularly when combined with a low BMI [6, 8], hypothesised to result from a greater risk of frailty, undernutrition and poor physical resilience within lean slow walkers [8]. These findings for cardiovascular disease may therefore extend into infectious disease, with self-reported slow walkers found to have a high risk of severe COVID-19 outcomes, even when weight status is normal.

The key strength of this study is the large contemporary population with linked COVID-19 data with access to measures of both BMI and walking pace. This study also has several important limitations. As testing in the UK has not been universal, it is not possible to determine overall exposure to or infection with SARS-CoV-2; linkage will therefore only highlight those that have developed severe disease or died from COVID-19. As such, the findings should be interpreted as the overall risk to censor date in the UK Biobank cohort. Nevertheless, the risk of severe COVID-19 with obesity reported in this study is in line with early findings of other studies [2, 14, 16, 17]. As this is an evolving pandemic, data should be interpreted in relation to the first wave of the pandemic in England. This analysis used a measure of self-reported walking pace. Whilst this has the advantage of highlighting the potential importance of a simple and easy-to-measure risk factor, there are also potential limitations. Namely, although self-reported walking pace has been shown to be associated with cardiorespiratory fitness within UK Biobank [6], it is subject to possible reporting bias. Given this and the observational design, no definitive causal conclusions can be derived from our results; nevertheless, our findings—-showing that self-reported slow walkers are at high risk across BMI categories-have potential prognostic relevance. Last, the risk factors included in this study, including comorbidities, were measured a decade before the pandemic. Although BMI and measures of physical fitness have been shown to be stable over time in adults [18, 19], values may be less stable in older age.

In conclusion, as many countries are now experiencing a second wave of SARS-CoV-2 infections, identifying individuals at greatest risk of developing severe disease is crucial. This study highlights BMI and walking pace as potential risk factors for severe COVID-19, with slow walkers in particular having a high-risk profile even when combined with normal weight statues. Ongoing public health and research surveillance studies should therefore consider incorporating simple measures of physical fitness in addition to BMI as potential risk predictors of COVID-19 outcomes.

Acknowledgements Data were analysed using UK Biobank application number 36371.
Author contributions Concept and design: TY, CR, FZ, KK, Acquisition, analysis, or interpretation of data: All authors. Drafting of the manuscript: TY. Critical revision of the manuscript for important intellectual content: All authors. Statistical analysis: TY. Statistical support: FZ.

\section{Compliance with ethical standards}

Conflict of interest Prof. Kamlesh Khunti is Chair of the UK SAGE subgroup on ethnicity and COVID-19. No other conflicts have been declared.

Financial support This research was supported by the National Institute for Health Research (NIHR) Leicester Biomedical Research Centre, the NIHR Applied Research Collaboration - East Midlands, and a grant from the UKRI-DHSC COVID-19 Rapid Response Rolling Call (MR/V020536/1). The funder had no role in the design and conduct of the study; collection, management, analysis, and interpretation of the data; preparation, review, or approval of the manuscript; and decision to submit the manuscript for publication.

Publisher's note Springer Nature remains neutral with regard to jurisdictional claims in published maps and institutional affiliations.

\section{References}

1. Yang J, Zheng Y, Gou X, Pu K, Chen Z, Guo Q, et al. Prevalence of comorbidities in the novel Wuhan coronavirus (COVID-19) infection: a systematic review and meta-analysis. Int J Infect Dis. 2020;94:91-95.

2. Seidu S, Gillies C, Zaccardi F, Kunutsor SK, Hartmann-Boyce J, Yates $\mathrm{T}$, et al. The impact of obesity on severe disease and mortality in people with SARS-CoV-2: a systematic review and meta-analysis. Endocrinol Diabetes Metab. 2020;14:e00176.

3. Michalakis K, Ilias I. SARS-CoV-2 infection and obesity: common inflammatory and metabolic aspects. Diabetes Metab Syndr. 2020;14:469-71.

4. Maltese G, Corsonello A, Di Rosa M, Soraci L, Vitale C, Corica F, et al. Frailty and COVID-19: a systematic scoping review. J Clin Med. 2020;9:2106.

5. Hewitt J, Carter B, Vilches-Moraga A, Quinn TJ, Braude P, Verduri A, et al. The effect of frailty on survival in patients with COVID-19 (COPE): a multicentre, European, observational cohort study. Lancet Public Health. 2020;5:e444-51.

6. Yates T, Zaccardi F, Dhalwani NN, Davies MJ, Bakrania K, CelisMorales CA, et al. Association of walking pace and handgrip strength with all-cause, cardiovascular, and cancer mortality: a UK Biobank observational study. Eur Heart J. 2017;14:3232-40.

7. Stamatakis E, Kelly P, Strain T, Murtagh EM, Ding D, Murphy MH. Self-rated walking pace and all-cause, cardiovascular disease and cancer mortality: individual participant pooled analysis of 50,225 walkers from 11 population British cohorts. Br J Sports Med. 2018;52:761-8.

8. Zaccardi F, Davies MJ, Khunti K, Yates T. Comparative relevance of physical fitness and adiposity on life expectancy: a UK biobank observational study. Mayo Clin Proc. 2019;94:985-94.

9. Zaccardi F, Franks PW, Dudbridge F, Davies MJ, Khunti K, Yates T. Mortality risk comparing walking pace to handgrip strength and a healthy lifestyle: a UK Biobank study. Eur J Prev Cardiol. 2019;12:2047487319885041.

10. Argyridou S, Zaccardi F, Davies MJ, Khunti K, Yates T. Walking pace improves all-cause and cardiovascular mortality risk 
prediction: a UK Biobank prognostic study. Eur J Prev Cardiol. 2019;7:2047487319887281.

11. Sudlow C, Gallacher J, Allen N, Beral V, Burton P, Danesh J, et al. UK Biobank: An Open Access Resource for Identifying the Causes of a Wide Range of Complex Diseases of Middle and Old Age. PLoS Med. 2015;12:e1001779. https://doi.org/10.1371/ journal.pmed.1001779.

12. Armstrong A, Rudkin JK, Allen N, Crook DW, Wilson DJ, Wyllie $\mathrm{DH}$, et al. Dynamic linkage of COVID-19 test results between Public Health England's Second Generation Surveillance System and UK Biobank. Microbial Genomics Online. 2020;6:mgen000397.

13. Yates T, Zaccardi F, Razieh C, Gillies CL, Rowlands A, Kloecker DE. et al. Framework to aid analysis and interpretation of ongoing COVID-19 research. Welcome Open Res.2020;5:208

14. Williamson EJ, Walker AJ, Bhaskaran K, Bacon S, Bates C, Morton CE, et al. Factors associated with COVID-19-related death using OpenSAFELY. Nature. 2020;584:430-6.
15. Kruglikov IL, Scherer PE. The role of adipocytes and adipocytelike cells in the severity of COVID-19 infections. Obesity. 2020;28:1187-90.

16. Lighter J, Phillips M, Hochman S, Sterling S, Johnson D, Francois F, et al. Obesity in patients younger than 60 years is a risk factor for Covid-19 hospital admission. Clin Infect Dis. 2020;71:896-897.

17. Simonnet A, Chetboun M, Poissy J, Raverdy V, Noulette J, Duhamel A, et al. High prevalence of obesity in severe acute respiratory syndrome coronavirus-2 (SARS-CoV-2) requiring invasive mechanical ventilation. Obesity. 2020;28:1195-1199.

18. Bayer O, Krüger H, Von Kries R, Toschke AM. Factors associated with tracking of BMI: a meta-regression analysis on BMI tracking. Obesity. 2011;19:1069-76.

19. Mertens E, Clarys P, Mullie P, Lefevre J, Charlier R, Knaeps S, et al. Stability of physical activity, fitness components and diet quality indices. Eur J Clin Nutr. 2017;71:519-24. 